\title{
OPTIMAL PRICING FOR AN ADVANCE SALES SYSTEM WITH PRICE AND WAITING TIME DEPENDENT DEMANDS
}

\author{
Peng-Sheng You \\ National Chiayi University
}

(Received September 6, 2006; Revised December 19, 2006)

\begin{abstract}
This paper investigates an advance sales system wherein a firm sells an inventory over a limited planning time interval. The firm divides the planning time interval into several reservation periods and delivers the reserved orders at the end of each reservation period. The demands are considered as price and waiting time dependent. In addition, the situation in which customers may cancel their orders before receiving them is also considered. Two continuous time models are developed and analyzed. The ratio of refund to sales price is assumed to be fixed in the first model while is assumed to be time-dependent in the second model. Solution procedures are developed for both models in order to determine the number of reservation periods, the optimal sales price, and order size.
\end{abstract}

Keywords: Inventory, price, cancellation, reservation, order

\section{Introduction}

In environments wherein services or products are popular or needed only for a short season, an organization which expects to make profit from selling such items may face problems such as the frequency of providing the goods or service to customers during the sales season, the replenishment quantity for each service or sales plan over the sales season and the sales price of the item.

For instance, a travel agent selling whale watching tours during the whale watching season may face the problem of determining the frequency of watching tours (i.e., the tour frequency during the season), the resource such as seat and lifesaving equipment needed to load potential customers on scheduled tours and the suitable price for each tour. A diving company may encounter problems like the frequency of diving trips in one sales season, the number of equipment needed to be reserved for each scheduled diving trip and the manner in which the rented or purchased resource is sold. In addition to the previous examples. Other season goods companies also divide their sales season into several sales periods over which the goods are sold in advance and at the end of which customers' orders are satisfied. These companies include moon cakes manufacturers and mullet roe firms. They also face the problems such as the delivery frequency in the sales season, and the number of goods needed to be ordered or produced for each delivery date.

Most tangible products can be used for a couple of times. Unlike tangible products, service or some perishable goods is available only on specified dates Weatherford and Bodily [17]. From a financial point of view, it is important to manage the supply and demand. That is, organizations should pay attention to the supply that should be prepared and the manner by which demand is influenced.

A literature which illustrates the determination of related order size is the news vendor 
problem. Here, the decision maker purchases season goods such as newspapers and fashion goods under conditions such as a single chance to replenish supply for future demand and inability to fulfill unexpectedly high demand $[4,5,7-10,14]$.

The conventional news vendor views price as an external parameter instead of a decision variable. However, many business practices found in service and manufacturing industries reveal that price is a good way to influence demand. Thus, many researchers have focused on inventory models with a price-dependent demand.

Petruzzi and Dada [15] investigated an inventory problem of simultaneously determining the order quantity and selling price. Building upon the work of Petruzzi and Dada [15], Agrawal and Seshadri [1] dealt with an inventory problem in which a risk-averse retailer aims to maximize total expected utility through determining an order quantity and selling price. Khouja [11] explored an inventory problem under the condition that multiple discounts can be used to sell excess inventory. The discounts are equally spaced in terms of price on the domain 0 and initial selling price. Shinn et al. [16] studied the problem of simultaneously determining the retail price and order size under the condition that delay in payments is order-size-dependent.

The phenomenon that services are produced and consumed simultaneously implies that unsold services are lost after a specified date. This leads many organizations to use advance sale systems to improve their utility rate and revenues [12]. That is, they ask customers to make reservations for their purchase. Research on reservation management can be found in the area of revenue management $[2,3,18]$. Example of such research are the literature reviews in Weatherford and Bodily [17] and McGill and Ryzin [13].

While reservation systems are employed, two factors may influence demand. One is the cancellation phenomenon and the other is the waiting time which is the time interval between making and receiving an order. The phenomenon that customers cancel their reservations may result in the loss of revenue if the penalty from customers cannot cover the lost profit and organizations cannot immediately replace the cancellation with an order from another customer. Thus, the phenomenon of cancellation cannot be ignored when the replenishment decision is made.

Moreover, if customers wait for a long period of time before receiving their orders, their subjective feeling may be influenced in a negative way since waiting time may be viewed as an extra charge to the orders. This phenomenon may lead to the situation wherein demand decreases as waiting time increases [6]. In this paper, we discuss the case in which a firm divides a fixed seasonal time interval into several equal time reservation periods. Customers are required to make reservation and receive their orders at the end of their reserved period. It is reasonable to conjecture that the longer the reservation period, the longer the average waiting time. In order to positively influence the customers' purchasing behavior, a firm can increase their delivery frequency such as fright frequency and tour frequency in order to shorten reservation period and increase demand.

However, as delivery frequency increases, set up costs such as fixed operating costs and replenishment costs increase. Thus, while taking the advantage of improving demand by increasing the delivery frequency, a firm should consider how to balance the tradeoff between the additional costs from the shortening waiting time and the lost sales from the decrease in demand caused by the waiting time.

The aforementioned literature has barely considered the above-mentioned situations wherein reserved customers may cancel their orders before receiving their orders and demand may be influenced by waiting time. Such phenomenon exists in practice. By incorporating this phenomenon, this paper addresses the simultaneous determination of capacity size, 
sales price and service frequency. The decision maker is assumed to be able to shift the demand rate by pricing policy. The purpose of this paper is to find the optimal decision for maximizing the total profit over a finite sales season.

\section{A Model with Time Independent Refund}

The following notation is used through this paper.

$L=$ the length of the planning horizon,

$n=$ the number of reservation periods, a decision variable,

$T=$ the length of a reservation period with $n T=L$,

$Q=$ a replenishment quantity made at the start of a reservation period,

$I(t)=$ available inventory at time $t$ of a reservation period (initially $I(0)=Q$ ),

$p=$ the sales price, a decision variable,

$d_{t}(p, T)=$ demand rate at time $t$ when the sale price and the length of a reservation period are set at $p$ and $T$, respectively

$\theta=$ the fraction of the number of cancellation,

$g=$ the ratio of refund to sales price,

$s=$ set up cost, and

$c=$ unit purchasing cost.

Now, we will develop the mathematical model for this problem. Consider a situation in which a firm sells a seasonal item over a finite sales season $L$. The time interval is divided into several reservation periods. All customers are required to make reservations for their purchase and their willingness to purchase the item are assumed to depend on the sales price and the waiting time. For obtaining qualitative insights without much analytical complexity, demands, for this item are assumed to linearly depend on price and waiting time, i.e.,

$$
d_{t}(p, T)=a-b_{1} p-b_{2}(T-t)
$$

where $a>0$ is a constant value and represents the mean demand rate, and $b_{1}>0$ and $b_{2}>0$ are constants and represent the price and waiting time sensitivities, respectively. Assume that their exist a maximum waiting time $T^{*}$ such that no customer is willing to purchase the item when the waiting time is large than $T^{*}$. We make a reasonable assumption that $a>b_{2} T^{*}$. The purpose of the firm is to maximize the total profit over the planning horizon through determining the number of reservation periods, replenishment quantity and sales price.

Let $n$ and $T$ denote the number of reservation periods and the length of a reservation period, respectively. Then, it follows that $T=L / n$. Postulate that at the start of a reservation period, an identical replenishment quantity $Q$ is made and the replenishment quantity is assumed to be arrival at the end of each reservation period. We assume that all reserved orders are satisfied immediately after the arrival of a replenishment quantity.

Since customers with reservations may cancel their reservations, we assume that reserved customers may cancel their reservations before receiving their orders. In practice, the cancellation fraction $\theta$ may be dependent on time. However, for obtaining qualitative insights without much analytical complexity, we assume that the cancellation fraction $\theta$ is independent of time and proportional to the number of reservation level. We assume that the ratio of refund to sales price is predetermined and denoted by $g$. Since, in practice, a refund is no larger than a sales price, we make a reasonable assumption that the ratio $g$ is smaller 
than one. The firm aims to maximize the total profit over the planning horizon through determining (1) the number of reservation periods, (2) the replenishment quantity $Q$ which is made at the start of each reservation period, and (3) the optimal price $p$.

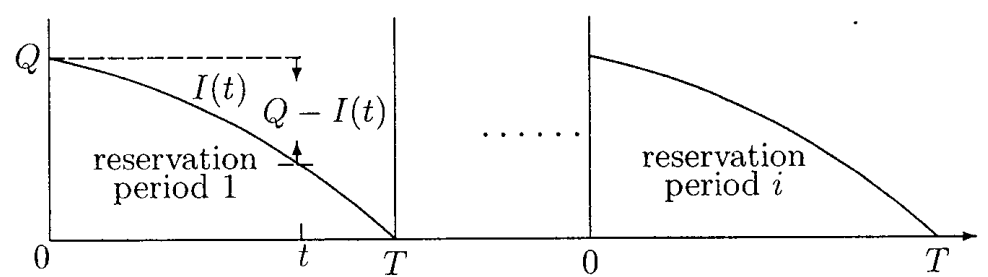

Figure 1: Available reservation level with time

Figure 1 depicts the available reservation level at any time during the planning horizon. Suppose the sales season is divided into $n$ reservation periods, each with time length $T=$ $L / n$. At time $t=0$ in a reservation period, a replenishment quantity $Q$ is made and the available reservation level is $I(0)=Q$. The available reservation level changes with time due to demands and cancellations. Since there is a demand at rate of $d_{t}(p, T)$ and a cancellation fraction of $\theta$, the available inventory level changes at a rate of $-d_{t}(p, T)+\theta(Q-I(t))$. Thus, the available inventory level of the system at any time of a reservation period can be described by the following differential equation.

$$
\frac{d I(t)}{d t}=-d_{t}(p, T)+\theta(Q-I(t)), \quad 0 \leq t \leq T .
$$

At the end of a reservation period $t=T$, the reservation level reaches $Q$ and thus the available reservation level reaches zero. At the same time, the replenishment quantity is arrival and all reservations are satisfied. Immediately after time $T$, another reservation period starts and the similar manner of the available reservation level repeats.

To develop our profit function, we will solve (2.2) to obtain reservation level function. Using the boundary conditions $I(0)=Q$, we obtain

$$
I(t)=Q-\frac{b_{2} t}{\theta}-\left(1-e^{-\theta t}\right)\left(\theta a-\theta b_{1} p-\theta b_{2} T-b_{2}\right) / \theta^{2} .
$$

Now, we will derive profit function. The components of profit function include the revenues from reservation, refund due to cancellation, purchasing cost and setup cost.

Revenues from sales: Let $R S$ denote revenues from sales over a reservation period. Then,

$$
R S=N_{s} p
$$

where $N_{s}$ represents the number of sales over a reservation period and is given by

$$
N_{s}=\int_{0}^{T} d_{t}(p, T) d t=-1 / 2 b_{2} T^{2}+a T-b_{1} p T .
$$

Refund from cancellation: In a reservation period, the total number of reservation at time $T$ is $Q$ and the total number of sales is $N_{s}$, thus the total number of cancellation $N_{c}$ in a reservation period is the difference between $N_{s}$ and $Q$. Consequently, refund from cancellation $C R$ over a reservation period is given by 


$$
C R=\left(N_{s}-Q\right) g p
$$

Ordering cost: Let $C P$ denote the total purchasing cost over a reservation period. Then,

$$
C P=Q c
$$

Objective function: Let $F(n, p, Q)$ denote the total revenues when the number of reservation periods, the sales price, replenishment quantity and are set at $n, p$ and $Q$, respectively. Then,

$$
F(n, p, Q)=n(R S-C R-C P-s) .
$$

Let $N_{\ell}$ denote the smallest $n$ such that $L / n<T^{*}$ and $N_{h}$ be the largest integer smaller than $L$. Then, our problem is to maximize the total profit function $F(n, p, Q)$ by determining the values of $n, p$ and $Q$ subject to the conditions of $N_{\ell} \leq n \leq N_{h}, T=L / n, Q \geq 0, p \geq 0$ and $d_{t}(p, T) \geq 0$ for all $t$. Using the boundary condition that $I(T)=0$ since the firm expects to sell out all of the replenishment at the end of a reservation period $T$, we obtain

$$
Q(n, p)=\frac{a \theta-b_{2}-b_{1} p \theta}{\theta^{2}}-\frac{1}{\theta^{2}} e^{(-\theta T)}\left(a \theta-b_{1} p \theta-b_{2} T \theta-b_{2}\right)
$$

Let $F(n, p)=F(n, p, Q(n, p))$. Then, we have

$$
F(n, p)=n\left(-K_{1} p^{2}+K_{2} p+K_{3} p+K_{4}\right)
$$

where

$$
\begin{aligned}
& K_{1}=b_{1}\left(g\left(1-e^{(-\theta T)}\right) / \theta+T(1-g)\right) \\
& K_{2}=0.5 T\left(2 a-b_{2} T\right)(1-g)+\left(1-e^{-\theta T}\right) b_{1} c / \theta \\
& K_{3}=g e^{-\theta T} b_{2} T / \theta+g a\left(1-e^{-\theta T}\right) / \theta-g b_{2}\left(1-e^{-\theta T}\right) / \theta^{2} \\
& K_{4}=-\left(1-e^{-\theta T}\right)\left(a \theta-b_{2}\right) c / \theta^{2}-e^{-\theta T} b_{2} T c / \theta-s .
\end{aligned}
$$

For fixed $n$, let the problem of $\max _{p} F(n, p)$ subject to the constraints of $p \geq 0, d_{t}(p, T) \geq$ 0 and $Q(n, p) \geq 0$ as problem $B_{n}$ and the corresponding optimal value as $F_{n}^{*}$. Then, the optimal profit of our problem can be found by $\max \left\{F_{N_{\ell}}^{*}, F_{N_{\ell}+1}^{*}, \cdots, F_{N_{h}}^{*}\right\}$. Below, we will develop some lemmas to solve problem $B_{n}$. First, for fixed $n$ let $\tilde{p}(n)$ denote the solution to the equation of $d F(n, p) / d p=n\left(-2 K_{1} p+K_{2}+K_{3}\right)=0$. Then, we have

$$
\tilde{p}(n)=\frac{K_{2}+K_{3}}{2 K_{1}}
$$

The value of $\tilde{p}(n)$ has the following characteristic.

Lemma 2.1. For $N_{\ell} \leq n \leq N_{h}, \tilde{p}(n)>0$. 
Proof. Note that $K_{1}>0$. Thus, if $K_{2}+K_{3}>0, \tilde{p}(n)>0$. Let $\beta(T)=K_{3}$. Then, since $\frac{d \beta(T)}{d T}=g e^{-\theta T}\left(a-b_{2} T\right)>0$ due to the modeling assumption of $a>b_{2} T^{*}>b_{2} T$ and that $\beta(T)=0$ when $T=0$, it follows that $\beta(T)>0$ for all $T$. Combining this and the fact that $K_{2}>0$, we have completed the proof.

For analyzing our model, we will re-format the constrains of $d_{t}(p, T) \geq 0$ and $Q(n, p) \geq 0$. For fixed $n$, let $p_{d}(n)$ be the solutions to the equation of $d_{0}(p, T)=0$. That is,

$$
p_{d}(n)=\frac{a-b_{2} T}{b_{1}}
$$

Then, since $d_{t}(p, T)$ is increasing in $t$ and decreasing in $p$, the constraint of $d_{t}(p, T) \geq 0$ can be replaced with $p \leq p_{d}(n)$. Let $p_{q}(n)$ be the solution to the equation of $Q(n, p)=0$. Than, we have

$$
p_{q}(n)=\frac{a \theta-b_{2}}{\theta b_{1}}+\frac{e^{-\theta T} b_{2} T}{b_{1}\left(1-e^{-\theta T}\right)} .
$$

Lemma 2.2. $Q(n, p)$ is decreasing in $p$.

Proof. Immediately from the fact that $\frac{d Q(n, p)}{d p}=-\frac{b_{1}\left(1-e^{-\theta T}\right)}{\theta}<0$.

Since $Q(n, p)$ is decreasing in $p$, the constraint of $Q(n, p) \geq 0$ can be replaced with the constraint of $p \leq p_{q}(n)$. Now, we will investigate the characteristics of $p_{q}(n)$ and $p_{q}(n)$.

Lemma 2.3. For $N_{\ell} \leq n \leq N_{h}, 0<p_{d}(n)<p_{q}(n)$.

Proof. The statement of $0<p_{d}(n)$ is immediately from the modeling assumption. The statement of $p_{d}(n)<p_{q}(n)$ is from the fact that $p_{d}(n)-p_{q}(n)=-b_{2}\left(\theta T-1+e^{-\theta T}\right) /\left(b_{1} \theta(1-\right.$ $\left.\left.e^{-\theta T}\right)\right)<0$. Thus we have completed the proof.

From Lemma 2.3, we see that a $p$ such as $0<p<p_{d}(n)$ satisfies the constraints of $p \geq 0$, $d_{t}(p, T) \geq 0$ and $Q(n, p) \geq 0$. Thus, the optimal sales price of problem $B_{n}$ must be selected from the set of $0<p<p_{d}(n)$.

Lemma 2.4. For fixed $n, F(n, p)$ is concave in $p$.

Proof. It is clear from the fact that $\frac{d^{2} F(n, p)}{d p^{2}}=-2 n K_{1}<0$ due to $K_{1}>0$.

Let $p(n)$ and $Q(n)$ respectively denote the optimal sales price and replenishment quantity when the planning horizon is divided into $n$ reservation periods. Then, we have the following theorem.

Theorem 2.1. The optimal values of $p(n)$ and $Q(n)$ are given by

$$
\begin{aligned}
p(n) & =\min \left\{\tilde{p}(n), p_{d}(n)\right\} \\
Q(n) & =\frac{1}{\theta^{2}}\left(a \theta-b_{2}-b_{1} p(n) \theta\right)-\frac{1}{\theta^{2}} e^{(-\theta T)}\left(a \theta-b_{1} p(n) \theta-b_{2} T \theta-b_{2}\right) .
\end{aligned}
$$

Proof. Equation (2.18) is clear from Lemma 2.1, Lemma 2.3 and Lemma 2.4. Equation (2.19) is clear from (2.9).

Let $n^{*}, p^{*}$ and $Q^{*}$ be the optimal number of reservation periods, sales price and replenishment quantity, respectively. Then, we can determine $n^{*}, p^{*}$ and $Q^{*}$ by the following procedure.

\section{Solution procedure I:}

1. let $n=N_{\ell}$ and $F^{*}=0$. 
2. computing $p(n), Q(n)$ by (2.18) and (2.19), respectively.

3. substituting $n, p(n)$ and $Q(n)$ into $F(n, p, Q)$ to obtain $F(n, p)$.

4. if $F^{*} \leq F(n, p)$, then let $n^{*}=n, p^{*}=p(n)$ and $Q^{*}=Q(n)$.

5. if $n<N_{h}$, then $n=n+1$ and return to step 2 .

6. print out the best solutions $n^{*}, p^{*}$ and $Q^{*}$.

Example 1: The preceding solution procedure can be illustrated by the following example. Suppose a season item such as mullet roe and lifesaving equipment for watching tours is needed over a sales season of $L=36$. A firm purchases or rents the item at a unit cost $c=10$ and resells it through reservation on the sales season. We assume that demands for this item is price and waiting time dependent and follows the function of $d_{t}(p, T)=$ $12-0.2 p-0.05(T-t)$.

We assume that the fraction of cancellation to the number of reserved order is assumed to be $\theta=0.01$. Additionally, the fraction of refund to sales price is assumed to be $g=0.7$. Finally, we assume that a set up cost $s=100$ is charged for each replenishment. The firm aims to maximize total profit by simultaneously determining (1) the number of reservation periods, (2) the sales price, and (3) the replenishment quantity.

Using the solution procedure I, we obtained optimal decision for each distinct number of reservation periods $n$. The computational results are described in Table 1 . Table 1 can be applied by the following example. Suppose the firm initially expects to delivery its goods to customers at the end of sales season. That is, the firm wants to set the number of reservation periods (delivery frequency) at one initially. In this situation, Table 1 will suggest the firm to set its order size at 140.3 and its sales price at $p(1)=\tilde{p}(1)=32.57$ since $\tilde{p}(1)=32.57<p_{d}(1)=51.0$. Under the suggestion, the firm will obtain the profit of 3308.5 when it sets its delivery frequency at one.

As can be shown from this table, the total revenues increases as the number of reservation periods increases. However, beyond a certain number, the total revenues decreases as the number of reservation periods increases. From this information, we see that the total profit of 3,796.2 is maximum when the number of reservation periods is set at $n^{*}=3$. Thus, the firm should set its delivery frequency at $n^{*}=3$ and set its sales price and order size for each reservation period at 34.17 and 55.1 , respectively

\section{A Model with Time Dependent Refund}

In this section, we analyze the case in which refund is time dependent. The notation used is the same as previous section. In addition, modeling assumptions are the same as section 2 except that the ratio of refund to sales price is assumed to be dependent on the time up to the deliver time point (the end of a reservation period) and to be the form of

$$
g(t, T)=(T-t) / T
$$

where $t$ is the time point at which cancellations occur and $T$ is the length of a reservation period. In this model, the derivatives of the reservation level $I(t)$, sales revenue $R C$ and purchasing cost $C P$ are the same as those in the previous model. However, the refund from cancellations is given by

$$
\hat{C R}=\int_{0}^{T} \theta I(t) g(t, T) p d t
$$


or equivalently,

$$
\begin{aligned}
\hat{C R}= & 0.5 \theta T p Q+b_{1}\left(1-\theta T+0.5 T^{2} \theta^{2}-e^{-\theta T}\right) p^{2} /\left(T \theta^{2}\right) \\
& -b_{2}\left(0.5 \theta^{2} T^{2}-1-T^{3} \theta^{3} / 3+(1+T \theta) e^{-\theta T}\right) p /\left(T \theta^{3}\right) \\
& -a\left(1-\theta T+0.5 \theta^{2} T^{2}-e^{-\theta T}\right) p /\left(\theta^{2} T\right)
\end{aligned}
$$

Let $V(n, p, Q)$ denote the total revenues when sales price, the replenishment quantity and the number of sales periods are set at $n, p$ and $Q$, respectively. Then,

$$
V(n, p, Q)=n(R S-\hat{C R}-C P-s)
$$

The purpose is to maximize the total profit $V(n, p, Q)$ by determining the values of $n$, $p$ and $Q$ subject to $N_{\ell} \leq n \leq N_{h}, n T=L, Q \geq 0, p \geq 0$ and $d_{t}(p, T) \geq 0$ for all $t$. Using the boundary condition that $I(T)=0$, we can observe that the optimal replenishment quantity $Q(n, p)$ is the same as that in $(2.9)$, the constraint of $Q(n, p) \geq 0$ can be replaced with $p \leq p_{q}(n)$ and the characteristics of $Q(n, p), p_{q}(n)$ and $p_{d}(n)$ are the same as those in Lemma 2.2 and Lemma 2.3, respectively. Let $V(n, p)=V(n, p, Q(n, p))$. Then, we have

$$
\begin{aligned}
V(n, p)= & -\frac{b_{1} w_{1}(\theta T) n}{2 \theta^{2} T} p^{2}+\frac{w_{1}(\theta T) n a}{2 \theta^{2} T} p+\frac{b_{1}\left(1-e^{-\theta T}\right) n c}{\theta} p-\frac{n b_{2} w_{2}(\theta T)}{6 \theta^{3} T} p \\
& -\frac{\left(a \theta+e^{-\theta T} b_{2} T \theta-b_{2}-a e^{-\theta T} \theta+b_{2} e^{-\theta T}\right) c n}{\theta^{2}}-n s
\end{aligned}
$$

where

$$
\begin{aligned}
& w_{1}(x)=2\left(x^{2}-x+1\right)+\left(x^{2}-2\right) e^{-x} \\
& w_{2}(x)=5 x^{3}-6 x^{2}+6+3\left(x^{3}+x^{2}-2 x-2\right) e^{-x}
\end{aligned}
$$

Lemma 3.1. For fixed $n, V(n, p)$ is concave in $p$.

Proof. Taking the first and second derivatives of $V(n, p)$ with respective to $p$ gives

$$
\begin{aligned}
\frac{\partial V(n, p)}{\partial p} & =-\frac{b_{1} w_{1}(\theta T) n}{\theta^{2} T} p+\frac{w_{1}(\theta T) n a}{2 \theta^{2} T}+\frac{b_{1}\left(1-e^{-\theta T}\right) n c}{\theta}-\frac{n b_{2} w_{2}(\theta T)}{6 \theta^{3} T} \\
\frac{\partial^{2} V(n, p)}{\partial p^{2}} & =-\frac{b_{1} w_{1}(\theta T) n}{\theta^{2} T}
\end{aligned}
$$

If $w_{1}(x) \geq 0$ for $x \geq 0$, the statement is proven. First, we have

$$
\begin{aligned}
\frac{d w_{1}(x)}{d x} & =2(2 x-1)-\left(x^{2}-2 x-2\right) e^{-x} \\
\frac{d^{2} w_{1}(x)}{d x^{2}} & =4+\left(x^{2}-4 x\right) e^{-x}
\end{aligned}
$$

Now, since $\frac{d^{2} w_{1}(x)}{d x^{2}}>0$ and $\left.\frac{d w_{1}(x)}{d x}\right|_{x=0}=0$, it follows that $\frac{d w_{1}(x)}{d x}>0$ for $x>0$. Note that $w_{1}(x)=0$ for $x=0$, thus $w_{1}(x)>0$ for $x>0$ and the result follows. 
Let $\hat{p}(n)$ denote the solution of $\frac{\partial V(n, p)}{\partial p}=0$. Then,

$$
\hat{p}(n)=0.5 a / b_{1}+c \theta T\left(1-e^{-\theta T}\right) / w_{1}(\theta T)-\frac{b_{2} w_{2}(\theta T)}{6 \theta w_{1}(\theta T) b_{1}} .
$$

Lemma 3.2. For $N_{\ell} \leq n \leq N_{h}, \hat{p}(n)>0$.

Proof. Since $a>b_{2} T^{*}>b_{2} T$ for $N_{\ell} \leq n \leq N_{h}$, we have

$$
\begin{aligned}
\hat{p}(n) & >0.5\left(b_{2} T\right) / b_{1}+c \theta T\left(1-e^{-\theta T}\right) / w_{1}(\theta T)-\frac{b_{2} w_{2}(\theta T)}{6 \theta w_{1}(\theta T) b_{1}} \\
& =\frac{b_{2} w_{3}(\theta T)}{6 \theta w_{1}(\theta T) b_{1}}+\frac{\left(1-e^{-\theta T}\right) c \theta T}{w_{1}(\theta T)}
\end{aligned}
$$

where

$$
w_{3}(x)=x^{3}+6 x-6-3\left(x^{2}-2\right) e^{-x} .
$$

In Lemma 3.1 we have shown that $w_{1}(x)>0$ for $x \geq 0$. Thus, $\hat{p}(n)>0$ if $w_{3}(x)>0$ for $x \geq 0$. Note $w_{3}(0)=0$. Thus, if $w_{3}(x)$ increases in $x, w_{3}(x)>0$ for $x>0$. It is observed that $\frac{d w_{3}(x)}{d x}=3 x^{2}+6+M$ where $M=3\left(x^{2}-2 x-2\right) e^{-x}$. Now, we will analyze the following two cases: Case of $\left(x^{2}-2 x-2\right)>=0$. In this case, it is clear that $\frac{d w_{3}(x)}{d x}>0$. Case of $\left(x^{2}-2 x-2\right)<0$. In this case, we have $\frac{d w_{3}(x)}{d x}>0$ due to $\frac{d w_{3}(x)}{d x}>3 x^{2}+6-6 x+3 x^{2}-6=6 x(x-1)>0$. Thus, $w_{3}(x)$ increases in $x$ and we have completed the proof.

Theorem 3.1. The optimal sales price of $p(n)$ is given by

$$
p(n)=\min \left\{\hat{p}(n), p_{d}(n)\right\},
$$

Proof. It can be proven by Lemma 3.1, Lemma 3.2 and Lemma 2.3.

The optimal value of $Q(n)$ is the same as the formula of equation (2.19). Using the similar procedure as that in solution procedure $I$, we can find the optimal sales price, the number of reservation periods and the replenishment quantity.

Example 2: In this example, the similar solution procedure is used to develop optimal decisions. The example used in this example is the same as that in example 1 except that the fraction of refund to sales price is assumed to follows the function $g(t, T)=(T-t) / T$.

The computational results are described in Table 2. The interpretations of Table 2 are similar to that of Table 1 . It can be noticed from this table that the total profit of 3806.8 is maximum when the number of reservation periods is set at $n=3$, and the optimal sales price and order size for each reservation period are 34.13 and 55.2, respectively.

\section{Conclusion}

This paper investigated a perishable inventory problem wherein a firm purchases and prices a service or a perishable item before a sales season and sells it through a reservation system during the sales season. Numerous models done in the past have addressed the perishable inventory problem. However, few have considered a situation wherein reserved customers are allowed to cancel their reservations and the backordered demands are waiting time dependent. Taking the cancellation phenomenon and the effect of waiting time on demand into 
account, this paper developed two continuous time models to respectively solve a problem with a time independent refund and a problem with a time dependent refund.

Analytic results revealed that the sales price and the replenishment quantity for each reservation period can be derived by a simple formula. From this result, the optimal decision was found by a simply solution procedure.

\section{Acknowledgement}

The authors would like to thank two anonymous referees for their helpful comments and suggestions that greatly improved the presentation of this paper. This research is partially supported by National Science Council, Taiwan, under grant NSC 95-2221-E-415-012-MY3.

\section{References}

[1] V. Agrawal and S. Seshadri: Impact of uncertainty and risk aversion on pricing and order quantity in the newsvendor problem. Manufacturing and Service Operations Management, 2 (2000), 410-423.

[2] M. Barut and V. Sridharan: Design and evaluation of a dynamic capacity apportionment procedure. European Journal of Operational Research, 155 (2004), 112-133.

[3] S.V. Boer, R. Freling and PN. iersma: Mathematical programming for network revenue management revisited. European Journal of Operational Research, 137 (2002), 72-92.

[4] S. Bose, A. Goswami and K.S. Chaudhuri: An EOQ Model for deteriorating items with linear time-dependent demand rate and shortages under inflation and time discounting. Journal of The Operational Research Society, 46 (1995), 771-782.

[5] K.J. Chung and S.F. Tsai: An algorithm to determine the EOQ for deteriorating items with shortage and a linear trend in demand. International Journal of Production Economics, 51 (1997), 215-225.

[6] H. Corsten and S. Stuhlmann: Capacity management in service organizations. Technovation, 18 (1998), 163-178.

[7] G. Gallego and I. Moon: The distribution free newsboy problem review and extensions. Journal of The Operational Research Society, 44 (1993), 825-834.

[8] Y. Gerchak and M. Parlar: A single period inventory problem with partially controllable demand. Computers and Operations Research, 14 (1987), 1-9.

[9] M.J. Khouja: Note on the newsboy problem with an emergency supply option. Journal of The Operational Research Society, 47 (1996), 1530-1534.

[10] M.J. Khouja: The single-period (news-vendor) problem: literature review and suggestions for future research. Omega-International Journal of Management Science, 27 (1999), 537--553.

[11] M.J. Khouja: Optimal ordering, discounting, and pricing in the single-period problem. Internal Journal of Production Economics, 65 (2000), 201-216.

[12] K.S. Lee and I.C.L. Ng: Advanced sale of service capacities: a theoretical analysis of the impact of price sensitivity on pricing and capacity allocations. European Journal of Operational Research, 54 (2001), 219-225.

[13] J.I. McGill and G.J.V. Ryzin: Revenue management: research overview and prospects. Transportance Science, 27 (1999), 252-265.

[14] I. Moon and S. Choi: The distribution free newsboy problem with balking. Journal of The Operational Research Society, 46 (1995), 537-542.

[15] N.C. Petruzzi and M. Dada: Pricing and the newsvendor problem: A review with extensions. Operations Research, 47 (1999), 183-194. 
[16] S.W. Shinn and H. Hwang: Optimal pricing and ordering policies for retailers under order-size-dependent delay in payments. Computers and Operations Research, 30 (2003), 35-50.

[17] L.R. Weatherford and S.E. Bodily: A taxonomy and research overview of perishableasset revenue management: Yield management, overbooking, and pricing. Operations Research, 40 (1992), 831--844.

[18] P.S. You: Dynamic pricing of inventory with cancellation demand. Journal of The Operational Research Society, 54 (2003), 1093-1101.

Peng-Sheng You

Graduate Institute of Transportation \& Logistics National Chiayi University

300 Shiue-Fu Road, Chiayi 600, TAIWAN

E-mail: psyuu@mail.ncyu.edu.tw 